\title{
The complete cd-index of dihedral and universal Coxeter groups
}

\author{
Saúl A. Blanco* \\ Department of Mathematics \\ Cornell University \\ Ithaca, NY 14853, USA \\ sabr@math.cornell.edu
}

Submitted: Jun 7, 2011; Accepted: Aug 15, 2011; Published: Sep 2, 2011

Mathematics Subject Classifications: 05E99, 20F55, 05E15

\begin{abstract}
We present a description, including a characterization, of the complete cd-index of dihedral intervals. Furthermore, we describe a method to compute the complete cd-index of intervals in universal Coxeter groups. To obtain such descriptions, we consider Bruhat intervals for which Björner and Wachs's CL-labeling can be extended to paths of different lengths in the Bruhat graph. While such an extension cannot be defined for all Bruhat intervals, it can be in dihedral and universal Coxeter systems.
\end{abstract}

\section{Introduction}

Let $(W, S)$ be a Coxeter system, and $u, v \in W$ with $u \leq v$ in Bruhat order. The Bruhat interval $[u, v]$ has been shown to be lexicographically shellable. This was proved by Björner and Wachs [3] and Dyer [11], in which the authors construct a CL-labeling and ELlabeling, respectively. Dyer's labeling can be given to $u-v$ paths of any length in the Bruhat graph $B(u, v)$ of $[u, v]$. However Björner and Wachs's labeling can only be used, in general, for the maximal-length paths. Nevertheless there are examples of Bruhat intervals in which both labeling procedures can be used for all $u-v$ paths. We call such intervals BW-labelable; for example, intervals in dihedral and universal Coxeter systems are BW-labelable. We show that both labelings (when defined) have the same descentset distribution. Thus one can compute the complete cd-index of Billera and Brenti [1] for such intervals utilizing the BW-labeling. The computation of the complete cd-index for intervals in universal Coxeter groups cannot be carried out in an easy way (if at all)

*Partially supported by NSF grant DMS-0555268 
utilizing reflection orders, as these orders are not easy to generate for infinite groups. Thus the BW-labeling is a tool that allows said computation for universal (and dihedral) Coxeter groups.

The paper is organized as follows. The basic definitions are given in Section 1.1. In Section 3 we provide a better description of the descent set distribution of $u-v$ paths in $B(u, v)$ when $[u, v]$ is a dihedral interval, i.e., an interval that is isomorphic to an interval in a dihedral group. This description is given in terms of the complete cd-index, and it is easily derived by using the BW-labeling discussed in Section 2. Furthermore, we show that the dihedral intervals are the only ones with complete cd-index containing only terms that are powers of $\mathbf{c}$. In Section 3.4 we show that among the $u-v$ paths of $B(u, v)$, the lexicographically-first ones are rising. This extends somewhat a result of Dyer $[11$, Proposition (4.3)].

\section{$1.1 \quad$ Basic definitions}

A Coxeter system is a pair $(W, S)$ where $W$ is a group with presentation $\left\langle S:\left(s_{i} s_{j}\right)^{m_{i, j}}=\right.$ $\left.\left(s_{j} s_{i}\right)^{m_{j, i}}=e\right\rangle$, where $m_{i, j} \in \mathbb{Z}_{>0} \cup\{\infty\}$ satisfies $m_{i, i}=1$ and if $i \neq j, m_{i, j}=m_{j, i}>2$ (possibly $\infty$ ). We call $W$ a Coxeter group and write $W=\left\langle S \mid\left(s_{i} s_{j}\right)^{m_{i, j}}=e\right\rangle$ instead of $\langle S$ : $\left.\left(s_{i} s_{j}\right)^{m_{i, j}}=\left(s_{j} s_{i}\right)^{m_{j, i}}=e\right\rangle$. An element $w$ of $W$ is of the form $s_{1} s_{2} \cdots s_{k}$, where each $s_{i} \in S$. The length $\ell(w)$ of $w$ is the minimal such $k$, and in this case we say that the expression $s_{1} s_{2} \cdots s_{k}$ is a reduced expression for $w$. As is customary we use $s_{1} s_{2} \cdots \widehat{s_{i}} \cdots s_{k}$ to denote the expression $s_{1} \cdots s_{i-1} s_{i+1} \cdots s_{k}$, i.e., $s_{1} s_{2} \cdots s_{k}$ with $s_{i}$ omitted. Two examples will be used constantly: the symmetric group $A_{n}$ generated by the $n$ adjacent transpositions $s_{i}=(i \quad i+1)$, for $1 \leq i \leq n$, and the dihedral group $I_{2}(m)=\left\langle a, b: a^{2}=b^{2}=(a b)^{m}=e\right\rangle$ of order $2 m$.

The set $S$ is called the set of simple reflections and $T=T(W, S) \stackrel{\text { def }}{=}\left\{w s w^{-1} \mid w \in\right.$ $W, s \in S\}$ is called the set of reflections of $(W, S)$. The Bruhat graph is the directed graph with vertex set $W$ and edge set $E(W, S)$, where $(u, v) \in E(W, S)$ if and only if there exists $t \in T$ so that $u t=v$ and $\ell(v)>\ell(u)$. We write $B(u, v)$ to denote the Bruhat graph corresponding the to interval $[u, v]$. Moreover, we write $B_{k}(u, v)$ to denote the set of $u-v$ paths in the Bruhat graph of length $k$ (here the length is given by the number of edges between $u$ and $v$ ). We remark that maximal chains in $[u, v]$ can be thought of as (undirected) maximal-length paths in $B(u, v)$.

We say that $x \leq y$ in Bruhat order if there is a directed $x-y$ path in the Bruhat graph. Furthermore, we say that $y$ covers $x$, denoted by $x \lessdot y$, if $x \leq y$ and $\ell(y)=\ell(x)+1$. The interval $[x, y]$ has very well known properties; for instance, it is an Eulerian and CohenMacaulay poset. More specifically, it is the face poset of a regular cell decomposition of a sphere (see [3], [11]).

For $w \in W$, we define the negative set of $w$, denoted by $N(w)$, to be the set of reflections that shorten the length of $w$, i.e., $N(w)=\{t \in T \mid \ell(w t)<\ell(w)\}$. It is well known (see [11]) that if $s_{1} s_{2} \cdots s_{k}$ is a reduced expression for $w$ then $N(w)=\left\{t_{1}, \ldots, t_{k}\right\}$, where $t_{i}=s_{k} \cdots s_{k-i+2} s_{k-i+1} s_{k-i+2} \cdots s_{k}$ for $i=1, \ldots, k$.

A reflection subgroup $W^{\prime}$ of $W$ is any subgroup generated by a subset of $T$. Reflection 
subgroups $W^{\prime}$ are Coxeter groups with simple reflection $S^{\prime}=\left\{t \in T: N(t) \cap W^{\prime}=\{t\}\right\}$, i.e., $\left(W^{\prime}, S^{\prime}\right)$ is a Coxeter system (see [8]). A reflection subgroup is said to be dihedral if $\left|S^{\prime}\right|=2$.

Let $\left(W^{\prime},\left\{t_{1}, t_{2}\right\}\right)$ be a Coxeter system with $W^{\prime}$ being a dihedral reflection subgroup of $W$. Dyer [11] showed the existence of linear orders $<_{T}$ on $T$ satisfying either $t_{1}<_{T}$ $t_{1} t_{2} t_{1}<_{T} t_{1} t_{2} t_{1} t_{2} t_{1}<_{T} \cdots<_{T} t_{2} t_{1} t_{2} t_{1} t_{2}<_{T} t_{2} t_{1} t_{2}<_{T} t_{2}$ or $t_{2}<_{T} t_{2} t_{2} t_{2}<_{T} t_{2} t_{1} t_{2} t_{1} t_{2}<_{T}$ $\cdots<_{T} t_{1} t_{2} t_{1} t_{2} t_{1}<_{T} t_{1} t_{2} t_{1}<_{T} t_{1}$. These linear orders are called reflection orders. Given a reflection order $<_{T}$, an initial section $A_{T}$ of $<_{T}$ is a subset of $T$ with $r<_{T} t$ for all $r \in A_{T}$ and $t \in T \backslash A_{T}$. Unless otherwise stated, $<_{T}$ will denote a generic reflection order.

Consider a reduced expression $w=s_{1} s_{2} \cdots s_{k-1} s_{k}$ for $w \in W$. Then we say that the total order $s_{k}<_{w} s_{k} s_{k-1} s_{k}<_{w} \ldots<_{w} s_{k} s_{k-1} \cdots s_{2} s_{1} s_{2} \cdots s_{k-1} s_{k}$ is induced by the reduced expression $s_{1} \cdots s_{k}$ of $w$. Dyer [11, Lemma (2.11)] showed that if $W$ is finite, then all reflection orders on $T$ are induced by a choice of reduced expression for $w_{0}^{W}$, the maximal-length word in $W$. In fact, any finite initial section of a reflection order is induced by a reduced expression for some $w \in W$.

For a path $\Delta \in B_{k}(u, v)$ denoted by the labels (given by reflections) of its edges $\left(t_{1}, t_{2}, \ldots, t_{k}\right)$ and reflection order $<_{T}$, the descent set of $\Delta$ is defined by $D(\Delta) \stackrel{\text { def }}{=}\{i \in$ $\left.[k-1] \mid t_{i+1}<_{T} t_{i}\right\}$. We say that $\Delta$ is rising if $D(\Delta)=\emptyset$, and falling if $D(\Delta)=$ $[k-1]$. Dyer showed that the reflection order is an EL-labeling for $[u, v]$, that is, every edge is labeled so that every subinterval of $[u, v]$ has a unique chain that is rising and lexicographically-first. The existence of this EL-labeling has been used to prove algebraic and topological properties of $[u, v]$ (see [11] for details).

We recall that a composition of a positive integer $n$ into $t$ parts is a finite sequence of positive integers $\alpha=\left(\alpha_{1}, \alpha_{2}, \ldots, \alpha_{t}\right)$ such that $\sum_{i=1}^{t} \alpha_{i}=n$. We write $\alpha \models n$ to mean that $\alpha$ is a composition of $n$. Given two compositions $\alpha=\left(\alpha_{1}, \ldots, \alpha_{r}\right)$ and $\beta=\left(\beta_{1}, \ldots, \beta_{s}\right)$ of $n$, we say that $\alpha$ refines $\beta$ if and only if there exist $1 \leq i_{1}<i_{2}<\cdots<i_{s-1}<r$ such that $\sum_{j=i_{k-1}+1}^{i_{k}} \alpha_{j}=\beta_{k}$ for $k=1, \ldots, s$. Here we define $i_{0}=0$ and $i_{s}=r$. If $\alpha$ refines $\beta$, we write $\alpha \preceq \beta$.

For $\Delta \in B_{k}(u, v)$, we define the descent composition of $\Delta$ to be the composition $\left(\alpha_{1}, \cdots, \alpha_{t}\right) \models k$ such that $\left\{\alpha_{1}, \alpha_{1}+\alpha_{2}, \ldots, \alpha_{1}+\alpha_{2}+\cdots+\alpha_{t-1}\right\}=D(\Delta)$. We denote the descent composition of $\Delta$ by $\mathcal{D}(\Delta)$. For $u, v \in W$ and $\alpha \models k$, let

$$
c_{\alpha}(u, v)=\left|\left\{\Delta \in B_{k}(u, v) \mid \alpha \preceq \mathcal{D}(\Delta)\right\}\right| .
$$

Notice that $D(\Delta)=\emptyset$ is equivalent to $\mathcal{D}(\Delta)$ having exactly one part.

We remark, in passing, that the numbers $c_{\alpha}(u, v)$ can be used to compute the KazhdanLusztig polynomial of $[u, v]$. Details can be found in [2].

The number of paths in $B_{k}(u, v)$ that are rising is counted by $c_{k}(u, v)$. In fact the $c_{k}(x, y)$ can be used to obtain all $c_{\alpha}(u, v)$, where $[x, y] \subset[u, v]$, due to the convolution-like formula

$$
c_{\alpha}(u, v)=\sum_{u \leq x_{1} \leq \cdots x_{n-1} \leq v} c_{\alpha_{1}}\left(u, x_{1}\right) c_{\alpha_{2}}\left(x_{1}, x_{2}\right) \cdots c_{\alpha_{n}}\left(x_{n-1}, v\right),
$$

where $\alpha=\left(\alpha_{1}, \ldots, \alpha_{n}\right)$ (see [2, Proposition 5.54]). It is shown in [6] that $c_{\alpha}(u, v)$ does not depend on the choice of reflection order. 


\section{Descent-set distribution of the BW-labeling and the reflection order}

We write $\Delta \in B(u, v)$ to indicate that $\Delta$ is a $u-v$ path in the Bruhat graph of $[u, v]$. As a convention, $\Delta$ can be written in two ways:

(i) $\left(a_{0}=u<a_{1}<\cdots<a_{k}=v\right)$, with $a_{i} \in W$, when we want to refer to the vertices of $\Delta$. If $\Delta$ is a maximal-length $u-v$ path, then we write $\left(u=a_{0} \lessdot a_{1} \lessdot \cdots \lessdot a_{\operatorname{rk}([u, v])}=v\right)$ to emphasize that the edges of $\Delta$ represent cover relations. In particular, an edge in $B(u, v)$ can be thought of as a path of length one, and so the edge between $w$ and $w_{1}$ with $\ell(w)<\ell\left(w_{1}\right)$ is denoted by $\left(w<w_{1}\right)$.

(ii) $\left(t_{1}, \ldots, t_{k}\right)$, with $t_{i} \in T$ and $a_{i-1} t_{i}=a_{i}, i=1, \ldots, k$, when we wish to refer to the edges that $\Delta$ traverses.

\subsection{Björner and Wachs's CL-labeling}

For this subsection, we set $n=\operatorname{rk}([u, v])$.

Björner and Wachs [3] defined a chain labeling on the edges of $B_{n}(u, v)$. The existence of such a labeling depends on the following well-known property of Coxeter groups.

Theorem 2.1 (Strong Exchange Condition, [12], Theorem 5.8). Let $s_{1} s_{2} \cdots s_{r}\left(s_{i} \in S\right.$ ) be an expression for $w$, not necessarily reduced. Suppose a reflection $t \in T$ satisfies $\ell(w t)<\ell(w)$. Then there is an index $i$ for which $w t=s_{1} \cdots \widehat{s}_{i} \cdots s_{r}$ (omitting $s_{i}$ ). Furthermore, if the expression for $w$ is reduced, then $i$ is unique.

Notice that once a reduced expression for $v$ has been chosen, say $v=s_{1} s_{2} \cdots s_{r}$, one can obtain a reduced expression for any word in a maximal-length path $\Delta \in B(u, v)$ (corresponding to a maximal chain in $[u, v]$ ) by simply removing generators from $s_{1} s_{2} \cdots s_{r}$. Thus one can label each edge of $\Delta$ with the index of the generator removed. This produces a $C L$-labeling for the maximal-length paths of $B(u, v)$. The technical definition of CL-labelings is presented in [3]. Roughly speaking, this is a labeling on chains of $[u, v]$ so that every sub-interval of $[u, v]$ has a unique rising chain that is lexicographically-first. Each edge receives a labeling that depends on the maximal-length $u-v$ path in which it belongs, and not on the edge itself. Björner and Wachs [3] studied this CL-labeling and applied it to derive properties of Bruhat intervals.

We now describe Björner and Wachs's CL-labeling. Let $\Delta=\left(x_{0}=u \lessdot x_{1} \lessdot \cdots \lessdot x_{n}=\right.$ $v$ ) be a maximal-length path of $B(u, v)$ and $s_{1} s_{2} \cdots s_{k}$ be a reduced expression for $v$. Theorem 2.1 guarantees the existence of a reduced expression for $x_{n-1}$ of the form $s_{1} \cdots \widehat{s}_{j_{n}} \cdots s_{k}$, where $j_{n}$ is unique. Given this reduced expression for $x_{n-1}$, the same theorem yields the existence of an index $j_{n-1}$ so that the removal of $s_{j_{n-1}}$ from $s_{1} \cdots \widehat{s}_{j_{n}} \cdots s_{k}$ is a reduced expression for $x_{n-2}$. Proceeding in this manner, there is a unique index $j_{i} \in[k]$ so that removing $s_{j_{i}}$ from the reduced expression for $x_{i}$ yields a reduced expression for $x_{i-1}$. Björner and Wachs's labeling associates $\Delta$ with $\left(\lambda_{1}(\Delta), \lambda_{2}(\Delta), \ldots, \lambda_{n}(\Delta)\right)$, where $\lambda_{i}(\Delta)=j_{i}$ 

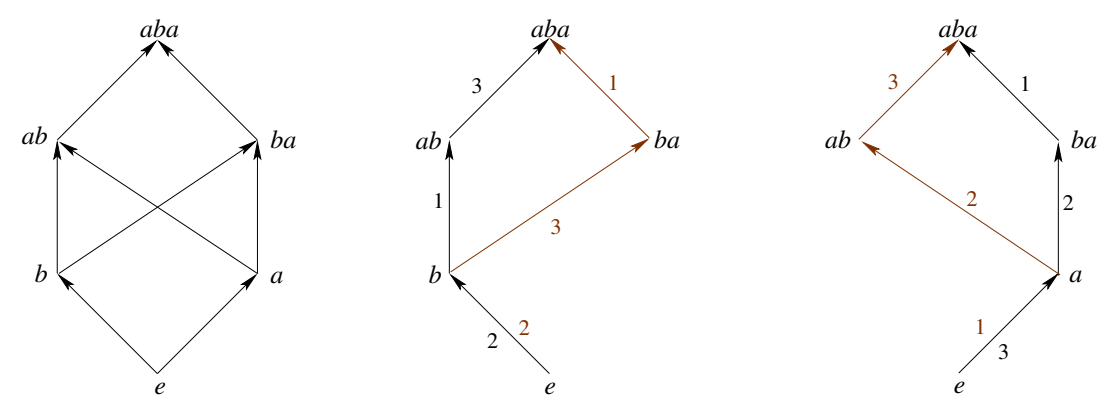

Figure 1: Björner and Wachs's labeling for maximal-length paths of $B(e, a b a)$. Notice that the edge $(e<a)$ has two possible labels depending on which " $a$ " is removed first from $a b a$.

To illustrate Björner and Wachs's CL-labeling, consider Figure 1. Notice that the labeling of the edge $(e \lessdot a)$ is either 1 or 3, depending on the index of the " $a$ " that is first removed. In general, if $\left(u=x_{0} \lessdot x_{1} \lessdot \cdots \lessdot x_{n}=v\right) \in B_{n}(u, v)$ is of maximal length, then the label of $\Delta_{1}=\left(u=x_{0} \lessdot x_{1} \lessdot \cdots \lessdot x_{j}\right)$ is uniquely determined once the label of $\Delta_{2}=\left(x_{j} \lessdot x_{j+1} \lessdot \cdots \lessdot x_{n}=v\right)$ has been chosen. In this situation we say that $\Delta_{1}$ is a rooted path; and more precisely, that $\Delta_{1}$ is rooted at $\Delta_{2}$.

In general, one cannot use Björner and Wachs's procedure to label paths $\Gamma=\left(x_{0}=\right.$ $\left.u<x_{1}<\cdots<x_{k}=v\right) \in B_{k}(u, v)$ if $k \neq n$. Indeed, by removing generators from a reduced expression for $v$ one could obtain a non-reduced expression for some $x_{i}$, and hence the index in Theorem 2.1 need not be unique. For example, consider the the non-reduced expression $s_{1} s_{2} s_{1} s_{2} s_{3} s_{2} s_{3} s_{2}$ for $s_{2} s_{1} s_{2} s_{3}$ in $A_{3}$. If one removes the first or fourth generator one obtains non-reduced expressions for $s_{2} s_{1} s_{3}$.

Nevertheless, there are cases where the Björner and Wachs's labeling procedure can be used to label all the edges of paths in $B(u, v)$. Some of these cases are discussed in the following subsection.

\subsection{BW-labelable Bruhat intervals}

We recall that the generators of a Coxeter system $(W, S)$ are subject to two types of relations, (cf. Section 3.3, [2]):

(i) nil relations, which are of the form $s^{2}=e$ for all $s \in S$, and

(ii) braid relations, which are of the form $\underbrace{s_{i} s_{j} s_{i} s_{j} \cdots}_{m_{i, j}}=\underbrace{s_{j} s_{i} s_{j} s_{i} \cdots}_{m_{i, j}}$ for all $s_{i}, s_{j} \in S, i \neq j$.

Definition 2.2. We say that an expression $s_{1} s_{2} \cdots s_{k}$ for $w \in W$ is nil-reduced if $s_{i} \neq s_{i+1}$ for $1 \leq i<k$.

Let $s_{1} s_{2} \cdots s_{n}$ be a nil-reduced expression for $v$. Given $A=\left\{i_{1}, \ldots, i_{j}\right\}$ we denote the expression $s_{1} \cdots \widehat{s}_{i_{1}} \cdots \widehat{s}_{i_{j}} \cdots s_{n}$ by $s_{[n] \backslash A}$. For any path $\Delta=\left(x_{0}=u<x_{1}<\right.$ $\left.\cdots<x_{k}=v\right) \in B_{k}(u, v)$, the Strong Exchange Condition gives the existence of sets $A_{k}(\Delta), A_{k-1}(\Delta), \ldots, A_{0}(\Delta) \subset[n]$ that are constructed recursively: $A_{k}(\Delta)=\emptyset$ and for 
$0 \leq i<k, b \in[n]$ is an element of $A_{i}(\Delta)$ if and only if there exists an expression for $x_{i}$ of the form $s_{[n] \backslash\left(\cup_{j>i}\left\{a_{j}\right\} \cup\{b\}\right)}$, where $a_{j} \in A_{j}(\Delta)$. Each $A_{i}(\Delta), 0 \leq i \leq k$, is called a removal set of $\Delta$. We remark that since the Björner and Wachs's procedure labels the edges from top to bottom, it is natural for our construction to start with $A_{k}$ and end with $A_{0}$.

Definition 2.3. We say that $[u, v]$ is $B W$-labelable if $\left|A_{i}(\Delta)\right|=1$ for all $k$ and $\Delta \in$ $B_{k}(u, v), 1 \leq i<k$. The $B W$-label of $\Delta$ is $\left(\lambda_{1}(\Delta), \ldots, \lambda_{k}(\Delta)\right)$, where $\left\{\lambda_{i}(\Delta)\right\}=A_{i}(\Delta)$. If every finite interval of a Coxeter group $W$ is BW-labelable, then we say that $W$ is BW-labelable. In other words, $[u, v]$ is BW-labelable if for all $\Delta \in B(u, v)$, the removal sets of $\Delta$ are singletons.

As an example, Figure 2 depicts the BW-labeling of $[e, a b a]$, where the interval is the full dihedral group of order 6 with generators $a, b$. Furthermore, Figure 3 shows the labels $(4,3,2,1)$ and $(1,3)$ that correspond to the paths $(e \lessdot a \lessdot b a \lessdot a b a \lessdot b a b a)$ and $(e<b<b a b a)$, respectively, where the intervals are in the dihedral group of order 8 with generators $a, b$.

Let $u \leq x \leq y \leq v$ be elements of $W$, with $[u, v]$ being BW-labelable, and consider a path $\Delta=\left(x_{0}=x<x_{1}<\cdots<x_{k}=y<\cdots<x_{k+m}=v\right) \in B_{k+m}(x, v)$. By the same reason as for maximal-length paths, the BW-label of $\Delta_{1}=\left(x_{0}=x<x_{1}<\cdots<x_{k}=\right.$ $y) \in B_{k}(x, y)$ depends on the BW-label of $\Delta_{2}=\left(x_{k}=y<\cdots<x_{k+m}=v\right) \in B_{m}(y, v)$. In this situation, we say that $\Delta_{1}$ is rooted at $\Delta_{2}$, or that $\Delta_{1}$ 's root is $\Delta_{2}$. Furthermore, once a reduced expression for $v$ has been fixed, the expressions for all the $x_{i}, 0 \leq i<k+m$ are completely determined as well. In this case, we say that the expressions obtained for the $x_{i}$ are given by following $\Delta$.

Remark 2.4. (i) Notice that the BW-label corresponding to paths $\Delta \in B_{\mathrm{rk}([u, v])}(u, v)$ is exactly the label assigned to $\Delta$ in Björner and Wachs's CL-labeling. In other words, the BW-label can always be given to $u-v$ paths of length $\operatorname{rk}([u, v])$.

(ii) Let $u_{1}, v_{1} \in W_{1}$ and $u_{2}, v_{2} \in W_{2}$, where $W_{1}, W_{2}$ are Coxeter groups. Suppose that $\left[u_{1}, v_{1}\right]$ and $\left[u_{2}, v_{2}\right]$ are BW-labelable, then $\left[u_{1}, v_{1}\right] \times\left[u_{2}, v_{2}\right]$ is a BW-labelable interval in $W_{1} \times W_{2}$. Indeed, the removal set of any path in $B\left(\left(u_{1}, u_{2}\right),\left(v_{1}, v_{2}\right)\right)$ is of the form $C \times D$, where $C$ is a removal set of a path in $B\left(u_{1}, v_{1}\right)$ and $D$ is a removal set of a path in $B\left(u_{2}, v_{2}\right)$.

Not all Bruhat intervals are BW-labelable, as can be seen in the example below.

Example 2.5. Consider the reduced expression $v=s_{1} s_{2} s_{1} s_{4} s_{2} s_{3} s_{2} s_{4} s_{3} s_{2} \in\left\langle s_{1}, \ldots, s_{4}\right.$ : $\left.s_{i}^{2}=\left(s_{1} s_{2}\right)^{3}=\left(s_{2} s_{3}\right)^{3}=\left(s_{1} s_{3}\right)^{2}=\left(s_{j} s_{4}\right)^{\infty}=e, i \in[4], j \in[3]\right\rangle$. Now consider $\Delta=$ $\left(u<s_{2} s_{1} s_{2} s_{3}<s_{2} s_{1} s_{3} s_{2} s_{4} s_{3} s_{2}<v\right) \in B_{3}(u, v)$, where $u=s_{2} s_{1} s_{3}$. Then $A_{3}(\Delta)=\emptyset$, $A_{2}(\Delta)=\{4\}$ (which corresponds the expression $s_{1} s_{2} s_{1} s_{2} s_{3} s_{2} s_{4} s_{3} s_{2}$ ), $A_{1}(\Delta)=\{8\}$ (which corresponds to the expression $s_{1} s_{2} s_{1} s_{2} s_{3} s_{2} s_{3} s_{2}$ ) and $A_{0}(\Delta)=\{1,5,9\}$ (which corresponds to the expressions $\left.s_{2} s_{1} s_{2} s_{3} s_{2} s_{3} s_{2}=s_{1} s_{2} s_{1} s_{3} s_{2} s_{3} s_{2}=s_{1} s_{2} s_{1} s_{2} s_{3}=u\right)$.

Thus $[u, v]$ is not BW-labelable. However, the groups of interest here, namely dihedral and universal Coxeter groups, are BW-labelable. We utilize this fact in Section 3. 


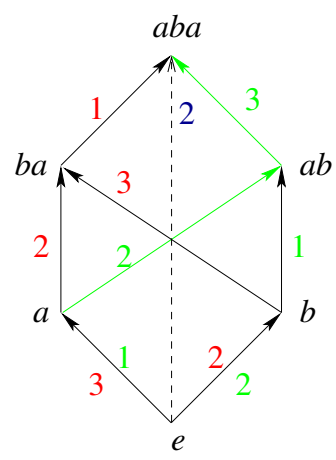

Figure 2: $[e, a b a]$ is BW-labelable. The path $(e<a b a)$ has label $\lambda_{1}((e<a b a))=2$.

We now present examples of BW-labelable intervals and groups.

Example 2.6. (a) If $B(u, v)$ has paths of exactly two different lengths then $[u, v]$ is BW-labelable. Indeed, the maximal-length $u-v$ paths can be labeled with Björner and Wachs's CL-labeling. Moreover, if $\Delta \in B_{\operatorname{rk}([u, v])-2}(u, v)$, then any expression for the vertices of $\Delta$ obtained by following $\Delta$ is either reduced or of the form $x s_{1} s_{2} s_{1} y$ with $\ell\left(x s_{1} \widehat{s}_{2} s_{1} y\right)=\ell(x y)=\ell\left(x s_{1} s_{2} s_{1} y\right)-3$. Thus the edge $\left(x y<x s_{1} s_{2} s_{1} y\right)$ has a unique label.

(b) Similarly, it can be argued that intervals $[u, v]$ of rank up to 5 are BW-labelable, since paths of length one correspond to the reflection $u^{-1} v$ and thus there is a unique label assigned to it (see the edge $(e<a b a)$ in Figure 2).

Example 2.7. Let $I_{2}(\infty)$ denotes the infinite dihedral group (which is the affine Weyl group $\left.\widetilde{A}_{1}\right)$. Let $[u, v]$ be an interval in $I_{2}(\infty)$ and $s_{1} \cdots s_{n}$ be a reduced expression for $v$. Since any nil-reduced expression for $x_{i}$ in $\Delta=\left(x_{0}=u<x_{1}<\cdots<x_{k}=v\right) \in B_{k}(u, v)$ obtained by removing generators of a reduced expression for $v$ is reduced, $[u, v]$ is BWlabelable. Indeed, the Strong Exchange Condition guarantees that $\left|A_{i}(\Delta)\right|=1$. So $I_{2}(\infty)$, and thus $I_{2}(n)$ for all $n \in \mathbb{Z}_{>0}$, is BW-labelable..

Example 2.8. One says that $(W, S)$ is universal if the only relation satisfied by $S$ are the nil-relations. Let $[u, v]$ be an interval in a universal Coxeter system and $s_{1} \cdots s_{n}$ be a reduced expression for $v$. Similar to the dihedral group case, any nil-reduced expression for an element in $[u, v]$ obtained by removing generators from $s_{1} \cdots s_{n}$ is reduced, and so $W$ is BW-labelable. This fact will allow us to compute the cd-index, as described in Section 3.

Definition 2.9. Let $[u, v]$ be a BW-labelable interval of a Coxeter system $(W, S), u \leq$ $x \leq y \leq v$ be elements of $W, \Gamma_{1}=\left(x_{0}=u<x_{1}<\cdots<x_{m}=x\right) \in B(u, x), \Delta=\left(x_{m}=\right.$ $\left.x<x_{m+1}<\cdots<x_{k}=y\right) \in B(x, y)$, and $\Gamma=\left(x_{k}=y<\cdots<x_{n}=v\right) \in B(y, v)$. Notice that $\Gamma$ is a root of $\Delta$.

(i) We denote the concatenation $\left(x_{0}=u<\cdots<x_{m}=x<\cdots<x_{k}=y<\cdots<\right.$ $\left.x_{n}=v\right)$ of $\Gamma_{1}, \Delta$ and $\Gamma$ by $\Gamma_{1} \Delta \Gamma$.

(ii) We define the $B W$-descent set of $\Delta$ with respect to $\Gamma, \Gamma_{1}$ as

$$
D_{\Gamma_{1}, \Gamma}^{B W}(\Delta) \stackrel{\text { def }}{=}\left\{i \in\{m+1, \ldots, k-1\} \mid \lambda_{i+1}\left(\Gamma_{1} \Delta \Gamma\right)<\lambda_{i}\left(\Gamma_{1} \Delta \Gamma\right)\right\} .
$$


Notice that the label given to $\Delta$ only depends on the choice of root $\Gamma$. So we drop $\Gamma_{1}$ from the notation and write simply $D_{\Gamma}^{B W}(\Delta)$.

(iii) We denote the $B W$-descent composition corresponding to $D_{\Gamma}^{B W}(\Delta)$ by $\mathcal{D}_{\Gamma}^{B W}(\Delta)$.

(iv) We say that $\Delta \in B(x, y)$ is $B W$-rising with respect to $\Gamma$ if $D_{\Gamma}^{B W}(\Delta)=\emptyset$. When $\Gamma$ is clear by the context, we simply write $B W$-rising.

(v) Define $c_{\alpha, \Gamma}^{B W}(x, y) \stackrel{\text { def }}{=}\left|\left\{\Delta \in B_{k}(x, y) \mid \alpha \preceq \mathcal{D}_{\Gamma}^{B W}(\Delta)\right\}\right|$, where $\alpha \models k$.

If $y=v$, then $\Gamma$ is the path with no edges. In this case, we ignore the reference to $\Gamma$ in the notation and write $D^{B W}(\Delta), \mathcal{D}^{B W}(\Delta)$ and $c_{\alpha}^{B W}(x, y)$, respectively.

We now prove that $c_{k, \Gamma}^{B W}(x, y)=c_{k}(x, y)$, for any $k \in \mathbb{Z}_{>0}, x, y$ and $\Gamma \in B(y, v)$ with $u \leq x \leq y \leq v$. This is the first step towards proving that $c_{\alpha, \Gamma}^{B W}(u, y)=c_{\alpha}(u, y)$.

Lemma 2.10. Let $[u, v]$ be a $B W$-labelable interval with $u \leq x \leq y \leq v$. Then for $k>0$, $c_{k, \Gamma}^{B W}(x, y)=c_{k}(x, y)$, regardless of the choice of $\Gamma \in B(y, v)$.

Proof. Let $s_{1} s_{2} \cdots s_{n}$ be the expression for $y$ given by following $\Gamma$. First let us assume that $s_{1} s_{2} \cdots s_{n}$ is reduced and let $C=\left(x_{0}=x<x_{1}<\cdots<x_{k}=y\right)$ be BW-rising. By the Strong Exchange Condition we have that $x=s_{1} \cdots \widehat{s_{1}} \cdots \widehat{s_{i_{k}}} \cdots s_{n}$. Since $C$ is BWrising, the BW-label associated to $C$ is $\left(i_{1}, i_{2}, \ldots, i_{k}\right)$ independently of the choice of $\Gamma$. Let $t_{j}=s_{n} \cdots s_{j+1} s_{j} s_{j+1} \cdots s_{n}$. Then $N(y)=\left\{t_{1}, \ldots, t_{n}\right\}$, and so $t_{n}<_{T} t_{n-1}<_{T} \cdots<_{T} t_{1}$ is the initial section for some reflection order $<_{T}$, by [11, Lemma (2.11), Proposition (2.13) and Remark 2.4(i)]. Since $y=x t_{i_{k}} \cdots t_{i_{1}}$, the label of $C$ with under $<_{T}$ is $\left(t_{i_{k}}, \ldots, t_{i_{1}}\right)$, and so $C$ is rising under $<_{T}$. It is easy to see that this construction is reversible, thus we have established a bijection between BW-rising paths and rising paths in the reflection order.

Now supposed that the expression $s_{1} s_{2} \cdots s_{n}$ for $y$ is not reduced, and let red $(y)$ be a reduced expression for $y$. Any BW-rising path in $B(x, \operatorname{red}(y))$, regardless of the choice of root, is obtained by removing generators of $\operatorname{red}(y)$ from right to left, and since $[u, v]$ is BW-labelable, there is a corresponding path in $B(x, y)$ whose BW-label is obtained by removing generators of the expression $s_{1} s_{2} \cdots s_{n}$ from right to left, and vice versa. Hence the number of BW-rising paths in $B\left(x, s_{1} s_{2} \cdots s_{n}\right.$ ) (the labels are given by rooting these paths at $\Gamma$ ) and $B(x, \operatorname{red}(y))$ (the labels are given by rooting at a maximal-length path of $B(y, v))$ is the same. Furthermore, by the argument made in the previous paragraph, the number of BW-rising paths in $[x, y]$ is the same as the number of rising paths in the reflection order.

Theorem 2.11. Let $[u, v]$ be a $B W$-labelable interval with $u \leq x \leq y \leq v$, and let $\alpha=\left(\alpha_{1}, \alpha_{2} \ldots, \alpha_{m}\right) \models k$ and $\Gamma \in B(y, v)$. Then $c_{\alpha, \Gamma}^{B W}(x, y)=c_{\alpha}(x, y)$.

Proof. We proceed by induction on $m$. If $m=1$, the statement follows from Lemma 2.10. If $m>1$, let $\widehat{\alpha}=\left(\alpha_{1}, \alpha_{2}, \ldots, \alpha_{m-1}\right)$. Then, 


$$
\begin{aligned}
c_{\alpha, \Gamma}^{B W}(x, y) & =\sum_{x \leq z \leq y} \sum_{\substack{\Delta \in B_{\alpha m}(z, y) \\
D_{\Gamma}^{B W}(\Delta)=\emptyset}} c_{\Delta \leq, \widehat{\alpha}}^{B W}(x, z) \\
& =\sum_{\substack { \Delta \leq z \leq y \\
\begin{subarray}{c}{\Delta \in B_{\alpha m}(z, y) \\
D_{\Gamma}^{B W}(\Delta)=\emptyset{ \Delta \leq z \leq y \\
\begin{subarray} { c } { \Delta \in B _ { \alpha m } ( z , y ) \\
D _ { \Gamma } ^ { B W } ( \Delta ) = \emptyset } }\end{subarray}} c_{\widehat{\alpha}}(x, z) \\
& =\sum_{x \leq z \leq y} c_{\widehat{\alpha}}(x, z) \sum_{\substack{\Delta \in B_{\alpha_{m}}(z, y) \\
D_{\Gamma}^{B W}(\Delta)=\emptyset}} 1 \\
& =\sum_{x \leq z \leq y} c_{\widehat{\alpha}}(x, z) c_{\alpha_{m}, \Gamma}^{B W}(z, y) \\
& =\sum_{x \leq z \leq y} c_{\widehat{\alpha}}(x, z) c_{\alpha_{m}}(z, y) \\
& =c_{\alpha}(x, y) .
\end{aligned}
$$

The second equality follows by induction and the last one from Lemma 2.10 and $(1.1)$.

In particular, if $[u, v]$ is BW-labelable then $c_{\alpha}^{B W}(u, v)=c_{\alpha}(u, v)$. Thus the BWlabeling and the reflection order yield the same descent-set distribution on the set of paths in $B(u, v)$.

Example 2.12. Consider the interval $\left[e, s_{2} s_{1} s_{3} s_{2} s_{1}\right]$ in $A_{3}$ (corresponding to [1234, 4312] in one-line notation for permutations). In particular the ten elements of $B_{3}\left(e, s_{2} s_{1} s_{3} s_{2} s_{1}\right)$. Using either the BW-labeling or the reflection order, the descent sets for these ten elements are: $\emptyset$ (two of them), $\{1\}$ (three of them), $\{2\}$ (three of them), and $\{1,2\}$ (two of them).

\section{Complete cd-index}

\subsection{Complete cd-index of Bruhat intervals}

Billera and Brenti [1] provided a way to encode the descents sets of paths in $B(u, v)$ with a non-homogeneous polynomial on the non-commutative variables $\mathbf{c}$ and $\mathbf{d}$. The encoding is done as follows: For a path $\Delta=\left(t_{1}, t_{2}, \ldots, t_{k}\right) \in B_{k}(u, v)$, let $w(\Delta)=x_{1} x_{2} \cdots x_{k-1}$, where $x_{i}=\mathbf{a}$ if $t_{i}<_{T} t_{i+1}$, and $x_{i}=\mathbf{b}$, otherwise. In other words, set $x_{i}$ to a if $i \notin D(\Delta)$ and to $\mathbf{b}$ if $i \in D(\Delta)$. Billera and Brenti also showed that $\widetilde{\Psi}_{u, v}(\mathbf{a}, \mathbf{b}) \stackrel{\text { def }}{=} \sum_{\Delta \in B(u, v)} w(\Delta)$ becomes a polynomial in the variables $\mathbf{c}$ and $\mathbf{d}$, where $\mathbf{c}=\mathbf{a}+\mathbf{b}$ and $\mathbf{d}=\mathbf{a b}+\mathbf{b a}$. This polynomial is called the complete $\mathbf{c d}-$ index of $[u, v]$, and it is denoted by $\widetilde{\psi}_{u, v}(\mathbf{c}, \mathbf{d})$. Notice that the complete cd-index of $[u, v]$ is an encoding of the distribution of the descent sets of each path $\Delta$ in the Bruhat graph of $[u, v]$, and thus seems to depend on $<_{T}$. However, it can be shown that this is not the case. For details on the complete cd-index, see [1]. 
The degree of a term in $\widetilde{\psi}_{u, v}(\mathbf{c}, \mathbf{d})$ is given by noticing that $\operatorname{deg}(\mathbf{c})=1$ and $\operatorname{deg}(\mathbf{d})=2$. For instance, $\operatorname{deg}\left(\mathbf{d}^{2} \mathbf{c}\right)=5$.

For example, consider $A_{2}$, the symmetric group on 3 elements with generators $s_{1}=$ $\left(\begin{array}{ll}1 & 2\end{array}\right)$ and $s_{2}=\left(\begin{array}{ll}2 & 3\end{array}\right)$. Then $t_{1}=s_{1}<_{T} t_{2}=s_{1} s_{2} s_{1}<_{T} t_{3}=s_{2}$ is a reflection order. The paths of length 3 are: $\left(t_{1}, t_{2}, t_{3}\right),\left(t_{1}, t_{3}, t_{1}\right),\left(t_{3}, t_{1}, t_{3}\right)$, and $\left(t_{3}, t_{2}, t_{1}\right)$, that encode to $\mathbf{a}^{\mathbf{2}}+\mathbf{a b}+\mathbf{b a}+\mathbf{b}^{\mathbf{2}}=\mathbf{c}^{\mathbf{2}}$. There is one path of length 1 , namely $t_{2}$, which encodes simply to 1 . So $\widetilde{\psi}_{u, v}(\mathbf{c}, \mathbf{d})=\mathbf{c}^{2}+1$.

We remark that $\left[9\right.$, Proposition (3.3)] shows that if $B_{k}(u, v) \neq \emptyset$ and $k \neq \operatorname{rk}([u, v])$ then $B_{k+2}(u, v) \neq \emptyset$. As a consequence, if $\widetilde{\psi}_{u, v}(\mathbf{c}, \mathbf{d})$ has terms of degree $k-1$ (corresponding to paths of length $k$ ), then it also has terms of degree $k+1$ (corresponding to paths of length $k+2$ ).

There are some specializations of the complete cd-index that count paths in $B(u, v)$. For instance, we have the lemma below.

Lemma 3.1. Let $[u, v]$ be a Bruhat interval. Then, (i) $\widetilde{\psi}_{u, v}(2,2)=|\{\Delta: \Delta \in B(u, v)\}|$, the number of paths of $B(u, v)$, and

(ii) $\widetilde{\psi}_{u, v}(1,0)=|\{\Delta \in B(u, v): D(\Delta)=\emptyset\}|$, the number of rising (or falling) paths of $B(u, v)$.

Proof. (i) To each path $\Delta \in B(u, v)$ there is a corresponding $w(\Delta)$ as defined at the beginning of this section. Hence, the number of ab-monomials, $\widetilde{\Psi}_{u, v}(1,1)$, equals the number of paths in $B(u, v)$. Since $\widetilde{\Psi}_{u, v}(1,1)=\widetilde{\psi}_{u, v}(2,2)$, we obtain the desired result.

(ii) By definition, $\widetilde{\Psi}_{u, v}(1,0)$ gives the number of rising paths of $B(u, v)$ and $\widetilde{\Psi}_{u, v}(0,1)$ gives the number of falling paths. Notice that $\widetilde{\Psi}_{u, v}(0,1)=\widetilde{\Psi}_{u, v}(1,0)=\widetilde{\psi}_{u, v}(1,0)$, and the result follows.

By [1, Theorem 2.2 and Corollary 2.3], it follows that $\widetilde{\psi}_{u, v}(\mathbf{c}, \mathbf{d})$ can be computed from the numbers $c_{\alpha}(u, v)$. Thus in view of Theorem 2.11, if $[u, v]$ is BW-labelable we can compute $\widetilde{\psi}_{u, v}(\mathbf{c}, \mathbf{d})$ using the identity $c_{\alpha}^{B W}(u, v)=c_{\alpha}(u, v)$. In the next two subsections, we use the BW-label to compute $\widetilde{\psi}_{u, v}(\mathbf{c}, \mathbf{d})$ for dihedral intervals and intervals in universal Coxeter groups.

\subsection{Dihedral intervals}

Let $u, v \in I_{2}(m)$ with $u \leq v$, then the isomorphism type of $B(u, v)$ is well known. For example, Figure 3 depicts $I_{2}(4)$.

Dyer [9] observed that if $W_{1}$ and $W_{2}$ are dihedral reflection subgroups and $W_{1} \cap$ $W_{2}$ contains a dihedral reflection subgroup $W_{3}$, then $\left\langle W_{1}, W_{2}\right\rangle$ is a dihedral reflection subgroup. This observation will be used in the proof of Lemma 3.2.

We say that a Bruhat interval $[u, v]$ is dihedral if it is isomorphic to an interval in a dihedral reflection subgroup. In this section, we compute the complete cd-index of dihedral intervals. The computation is simplified if the BW-labeling is utilized, and so we take this approach. It turns out that it is enough to consider the case where $[u, v] \in I_{2}(m)$ for some $m$. We make this explicit in the following lemma. 
Lemma 3.2. Let $[u, v]$ be a dihedral interval of $(W, S)$, where the edges of $B(u, v)$ have been labeled by reflections. Then $\widetilde{\psi}_{u, v}(\boldsymbol{c}, \boldsymbol{d})=\widetilde{\psi}_{w, z}(\boldsymbol{c}, \boldsymbol{d})$, where $[w, z] \subset I_{2}(m)$ for some $m$.

Proof. Let $[u, v]$ be a dihedral interval in $B(W)$ and let $t_{1}, t_{2}, \ldots, t_{m}$ be all the reflections that correspond to the labels of the edges of $B(u, v)$. Let $w_{1}, w_{2}, w_{1}^{\prime}, w_{2}^{\prime} \in[u, v]$ with $u \lessdot w_{1}, u \lessdot w_{2}, w_{1}, w_{2} \lessdot w_{1}^{\prime}$, and $w_{1}, w_{2} \lessdot w_{2}^{\prime}$. Suppose that the labels of $B\left(u, w_{1}^{\prime}\right)$ and $B\left(u, w_{2}^{\prime}\right)$ are $t_{1}, t_{2}, t_{3}, t_{4}$ and $t_{1}, t_{2}, t_{5}, t_{6}$, respectively (see figure below). From Dyer [9, Lemma (3.1)], we have that $W_{1} \stackrel{\text { def }}{=}\left\langle t_{1}, t_{2}, t_{3}, t_{4}\right\rangle$ and $W_{2} \stackrel{\text { def }}{=}\left\langle t_{1}, t_{2}, t_{5}, t_{6}\right\rangle$ are dihedral reflection subgroups of $(W, S)$. Moreover, since $\left\langle t_{1}, t_{2}\right\rangle \subset W_{1} \cap W_{2}$, then $\left\langle t_{1}, t_{2}, \ldots, t_{6}\right\rangle$ is a dihedral reflection subgroup of $(W, S)$. Proceeding in a similar manner, we conclude that $W^{\prime} \stackrel{\text { def }}{=}\left\langle t_{1}, t_{2}, \ldots, t_{m}\right\rangle$ is a dihedral reflection subgroup of $(W, S)$.

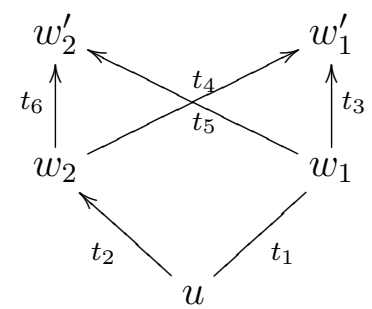

By [9, Theorem (1.4)(ii)] and [10, Proposition 1.4(3)], there exists a label-preserving isomorphism (the labels are given by reflections) between $B_{W}\left(W^{\prime}\right)$ and $B_{W}\left(W^{\prime} u\right)$, where $B_{W}(A)$ denotes the induced subgraph of $B(W)$ with vertex set $A \subset W$. Notice that $B(u, v)$ is an induced subgraph of $B_{W}\left(W^{\prime} u\right)$. Furthermore, $B_{W}\left(W^{\prime}\right)=B\left(W^{\prime}\right)$ by $[9$, Theorem (1.4)(i)] and $B\left(W^{\prime}\right) \cong B\left(I_{2}(m)\right)$ as directed graphs, for some $m \in \mathbb{Z}_{>0} \cup\{\infty\}$. Now the result follows by observing that $B\left(W^{\prime}\right)$ and $B\left(I_{2}(m)\right)$ have the same descent-set distribution in any reflection order.

The previous result gives that $\widetilde{\psi}_{u, v}(\mathbf{c}, \mathbf{d})=\widetilde{\psi}_{w, z}(\mathbf{c}, \mathbf{d})$ and $\operatorname{rk}([w, z])=\operatorname{rk}([u, v])$, where $[w, z] \subset I_{2}(m)$ for some $m$. On the other hand, since $[w, z]$ is BW-labelable, Theorem 2.11 gives that we can compute $\widetilde{\psi}_{w, z}(\mathbf{c}, \mathbf{d})$ utilizing the BW-labeling. As it turns out, using the BW-labeling facilitates the computation.

We now describe the complete cd-index for dihedral intervals in terms of the $q$ Fibonacci polynomial of degree $n$, where $F_{n}(q)$ is defined by $F_{1}(q)=1, F_{2}(q)=q$, and $F_{n}(q)=q F_{n-1}(q)+F_{n-2}(q)$ for $n>2$.

Proposition 3.3. If $[u, v]$ is a dihedral interval of rank $n$, then $\widetilde{\psi}_{u, v}(\boldsymbol{c}, \boldsymbol{d})=F_{n}(\boldsymbol{c})$.

Proof. Lemma 3.2 gives that it is enough to consider the case $[u, v] \in I_{2}(m)$ for some $m$. Moreover, we can assume that paths in $[u, v]$ are labeled with the BW-labeling.

We proceed by induction on $n \stackrel{\text { def }}{=} \operatorname{rk}([u, v])$. If $n=1$ or $n=2$, it is easy to verify that the result holds.

Let $v_{1}, v_{2}$ be the two elements of rank $n-1$ in $[u, v]$. Notice that one of these, say $v_{1}$, is obtained from the chosen reduced expression of $v$ by removing the last generator. 


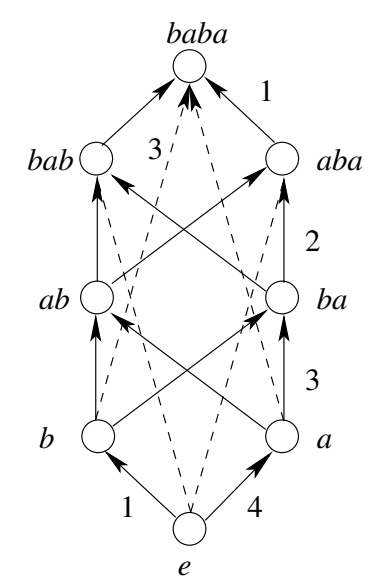

Figure 3: $B(u, v)$ with $u=e, v=b a b a=a b a b$ with $u, v \in I_{2}(4)$.

Thus for any path $\Delta=\left(u<\cdots<v_{1}<v\right) \in B_{k}(u, v)$, we have that $\lambda_{k-1}(\Delta)<$ $\lambda_{k}(\Delta)$. Hence the contribution of the $u-v$ paths through $v_{1}$ is $\mathbf{a} \widetilde{\psi}_{u, v_{1}}(\mathbf{c}, \mathbf{d})$. Similarly, $v_{2}$ is obtained from $v$ by removing the first generator of the reduced expression chosen for $v$. In this case the contribution of all paths $\Gamma=\left(u<\cdots<v_{2}<v\right)$ is $\mathbf{b} \widetilde{\psi}_{u, v_{2}}(\mathbf{c}, \mathbf{d})$, for $\lambda_{k}(\Gamma)<\lambda_{k-1}(\Gamma)$. Therefore, the contribution of all $u-v$ passing through $v_{1}$ or $v_{2}$ is $\mathbf{a} \widetilde{\psi}_{u, v_{1}}(\mathbf{c}, \mathbf{d})+\mathbf{b} \widetilde{\psi}_{u, v_{2}}(\mathbf{c}, \mathbf{d})=\mathbf{a} F_{n-1}(\mathbf{c})+\mathbf{b} F_{n-1}(\mathbf{c})=\mathbf{c} F_{n-1}(\mathbf{c})$

We are left with computing the contribution of paths that do not go through the vertices of rank $n-1$. Notice that an expression for each of the vertices in these paths is obtained from the reduced expression of $v$ by removing generators other than the first or last one. Thus the descent-set distribution of the paths in $B(u, v)$ that do not go through $v_{1}$ or $v_{2}$ is the same as that of an interval of the form $[u, x]$ with $\operatorname{rk}([u, x])=n-2$. Hence, the contribution of these paths is $\widetilde{\psi}_{u, x}(\mathbf{c}, \mathbf{d})=F_{n-2}(\mathbf{c})$, and the result follows.

Proposition 3.3 shows that the coefficients of $\widetilde{\psi}_{u, v}(\mathbf{c}, \mathbf{d})$, when $[u, v]$ is dihedral, are both non-negative and combinatorially invariant, i.e., only depend on the isomorphism type of $[u, v]$. Non-negativity and combinatorial invariance are conjectured for $\widetilde{\psi}_{u, v}(\mathbf{c}, \mathbf{d})$ in general (cf. [1, Conjecture 6.1 and Remark 4.13]). An enumerative consequence follows immediately by from Lemma 3.1 and setting $\mathbf{c}=1$ in $F_{n}(\mathbf{c})$.

Corollary 3.4. If $[u, v]$ is a dihedral interval of rank $n$, then the number of $u$ - $v$ rising (or falling) paths in $B(u, v)$ is the $n$-th Fibonacci number.

The following theorem yields that dihedral intervals are the only ones that do not contain a $\mathbf{d}$ in their complete $\mathbf{c d}$-index.

Theorem 3.5. Let $[u, v]$ be a Bruhat interval. Then $\widetilde{\psi}_{u, v}(\boldsymbol{c}, \boldsymbol{d})=\widetilde{\psi}_{u, v}(\boldsymbol{c}, 0)$ if and only if $[u, v]$ is a dihedral interval.

Proof. The theorem is vacuously true if the rank of $[u, v]$ is 1 , so we can assume that $\operatorname{rk}[u, v]>1$. If $[u, v]$ is dihedral, Proposition 3.3 gives that $\widetilde{\psi}_{u, v}(\mathbf{c}, \mathbf{d})=F_{\operatorname{rk}([u, v])}(\mathbf{c})=$ $\widetilde{\psi}_{u, v}(\mathbf{c}, 0)$. 
Suppose that $[u, v]$ is not dihedral. We show by contradiction that among the highestdegree terms of $\widetilde{\psi}_{u, v}(\mathbf{c}, \mathbf{d})$ there must be a term containing a $\mathbf{d}$. Let $p_{k}(u, v)$ be the number of paths of length $k$ from $u$ to $v$, and define $n \stackrel{\text { def }}{=} \operatorname{rk}([u, v])-1$. Since $[u, v]$ is Eulerian, any element $w \in[u, v)$ has at least two covers, and so there are at least two elements in each rank, except for the top and bottom elements. Moreover, since $[u, v]$ is not dihedral there are at least three elements of rank 1 , and so $p_{n+1}(u, v) \geq 3 \cdot 2^{n-1}$. Nevertheless, $[1$, Proposition 5.3] states that

$$
p_{n+1}(u, v)=\sum_{w: \operatorname{deg}(w)=n} 2^{n-|w|_{\mathbf{d}}}[w]_{u, v},
$$

where $|w|_{\mathbf{d}}$ is the number of d's in a cd-word $w$ and $[w]_{u, v}$ is the coefficient of $w$ in $\widetilde{\psi}_{u, v}(\mathbf{c}, \mathbf{d})$. Furthermore, if there are no cd-terms containing a $\mathbf{d}$ then there is a unique cd-word of degree $n$ which corresponds to the unique rising maximal-length path of length $n+1$. Hence $p_{n+1}(u, v)=2^{n}$. This contradicts $p_{n+1}(u, v)>2^{n}$, and the result follows.

\subsection{Universal Coxeter groups}

Reflection orders are easy to understand for any finite Coxeter group $W$. Indeed, they are all induced by a choice of reduced expression for $w_{0}^{W}$, the longest element of $W$ (see [11]). Furthermore, there are combinatorial descriptions of reflection orders for types $A$ and $B$. For instance, for type $A$, a reflection order is given by ordering the transpositions in lexicographic order (see [2]). A description for groups of type $B$ in terms of signed permutations can be found in [5]. Thus the complete cd-index for intervals in finite Coxeter groups can be easily computed. On the other hand, there is no known method to generate reflection orders for infinite Coxeter groups, not even in the "simple" case of universal Coxeter groups where there are no braid relations. The lack of such a method makes the computation of the complete cd-index extremely difficult (if not impossible). Fortunately, the BW-labeling allows us to compute $\widetilde{\psi}_{u, v}(\mathbf{c}, \mathbf{d})$ for intervals $[u, v]$ in universal Coxeter groups.

In general, the number reflections used to label paths in $B(u, v)$ is not given by the length of $v$. As an example, consider the interval $\left[e, s_{1} s_{2} s_{1} s_{3} s_{2}\right]$ of $A_{3}$, where $s_{1} s_{2} s_{1} s_{3} s_{2}$ is a reduced expression for the permutation 4312. There are five reflections induced by $s_{1} s_{2} s_{1} s_{3} s_{2}$, but the reflection order utilizes six reflections when labeling the edges of $B\left(e, s_{1} s_{2} s_{1} s_{3} s_{2}\right)$. Hence, knowing a reduced expression for $w \in W$ does not determine the number of reflections used to label the edges of paths in $B\left(e, s_{1} s_{2} s_{1} s_{3} s_{2}\right)$. This is not the case for the BW-labeling. Indeed, if $[u, v]$ is BW-labelable, all one needs to compute $D(\Delta)$ for $\Delta \in B(u, v)$ is a reduced expression for $v$. In particular, even if $|T(W, S)|$ is infinite (as is the case for universal Coxeter groups), the labels needed to compute the descent sets of paths in $B(u, v)$ are contained in the set $\{1,2, \ldots, \ell(v)\}$.

Let us illustrate a computation of the complete cd-index for an interval in a universal Coxeter group. 
Example 3.6. Consider the universal Coxeter group $W=\left\langle s_{1}, s_{2}, s_{3}: s_{1}^{2}=s_{2}^{2}=s_{3}^{2}=e\right\rangle$ and let $v=s_{2} s_{1} s_{2} s_{3} s_{1}$. Using the BW-labeling we obtain that the degree-two part of $\widetilde{\psi}_{e, v}(\mathbf{c}, \mathbf{d})$ are $2 \mathbf{c}^{2}+\mathbf{d}$. Notice that $v$ induces a reflection order $<_{T}$ with initial section

$$
s_{1}<_{T} s_{1} s_{3} s_{1}<_{T} s_{1} s_{3} s_{2} s_{3} s_{2}<_{T} s_{1} s_{3} s_{2} s_{1} s_{2} s_{3} s_{1}<_{T} s_{1} s_{3} s_{2} s_{1} s_{2} s_{1} s_{2} s_{3} s_{1}
$$

However, this initial section does not suffice to compute $\widetilde{\psi}_{e, v}(\mathbf{c}, \mathbf{d})$ using the reflection order, as some of the edges of paths in $B_{3}(e, v)$ are labeled with reflections that do not appear in that initial section. For example, the edge $\left(e<s_{2}\right)$ is labeled with $s_{2}$ and the edge $\left(s_{1} s_{3}<s_{1} s_{2} s_{3}\right)$ is labeled with $s_{3} s_{2} s_{3}$. Thus one cannot compute $\widetilde{\psi}_{e, v}(\mathbf{c}, \mathbf{d})$ with a reflection order using information contained in $[u, v]$ (at least using reduced expressions of elements in $[u, v]$ ).

\subsection{Existence of rising paths}

Let $[u, v]$ have rank $n$, then it follows from [9, Proposition (3.3)] that if $m<n$ and $B_{m}(u, v) \neq \emptyset$, then $B_{m+2}(u, v) \neq \emptyset$. So the lengths of paths in $B(u, v)$ increase by two and include all numbers congruent to $n$ modulo 2 between the smallest number $m$ such that $B_{m}(u, v) \neq \emptyset$ and $n$.

As we pointed out, it is shown in [11] that there is a unique rising path in $B_{\operatorname{rk}([u, v])}(u, v)$ that is lexicographically-first in the reflection order. In this subsection we show that the lexicographically-first path in $B_{k}(u, v) \neq \emptyset$, where $k \equiv \operatorname{rk}([u, v])(\bmod 2)$, is rising. In general, there might be more than one rising path.

The following theorem will be utilized in our proof of Proposition 3.8.

Theorem 3.7 ([7], Theorem 1). Let $(W, S)$ be a finite or affine Coxeter group and let $<_{T}$ be a reflection order for $W$. If $\left\{t_{1}, t_{2}, \ldots, t_{k}\right\} \subset T(W, S)$ and $t_{1}<_{T} t_{2}<_{T} \cdots<_{T} t_{k}$ then $t_{1} t_{2} \cdots t_{k} \neq e$.

We now follow [1] and define the flip of $\Gamma \in B_{2}(u, v)$. Let $\left(t_{1}, t_{2}\right),\left(r_{1}, r_{2}\right)$ be two distinct elements of $B_{2}(u, v)$, then we say that $\left(t_{1}, t_{2}\right) \leq_{l e x}\left(r_{1}, r_{2}\right)$ if $t_{1}<_{T} r_{1}$ or if $t_{1}=r_{1}$ and $t_{2}<_{T} r_{2}$. The existence of the complete $\mathbf{c d}$-index implies that there are as many paths with empty descent set in $B_{2}(u, v)$ as those with descent set $\{1\}$. Order all the paths in $B_{2}(u, v)$ using $\leq_{l e x}$ and let

$$
r(\Gamma)=\left|\left\{\Delta \in B_{2}(u, v) \mid D(\Delta)=D(\Gamma), \Delta \leq_{\text {lex }} \Gamma\right\}\right| .
$$

The flip of $\Gamma$ is the $r(\Gamma)$-th Bruhat path in $\left\{\Delta \in B_{2}(u, v) \mid D(\Delta) \neq D(\Gamma)\right\}$ ordered by $\leq_{\text {lex }}$. We denote this path by flip $(\Gamma)$.

The following was proved in the case of finite Coxeter and affine Weyl groups [1, Proposition 6.2]. We prove that the results holds for an arbitrary Coxeter group.

Proposition 3.8. Let $W$ be a Coxeter group, and let $u, v \in W, u<v,(u<y<v) \in$ $B_{2}(u, v)$ be such that $D((u<y<v))=\emptyset$ and $(u<x<v) \stackrel{\text { def }}{=}$ flip $((u<y<v))$. Then $u^{-1} y<_{T} u^{-1} x$ and $x^{-1} v<_{T} y^{-1} v$ for any reflection order $<_{T}$. 
Proof. Let $t_{1}=u^{-1} y, t_{2}=y^{-1} v, t_{3}=u^{-1} x$, and $t_{4}=x^{-1} v$. Notice that the reflection subgroup $W^{\prime}=\left\langle t_{1}, t_{2}, t_{3}, t_{4}\right\rangle$ is dihedral, by [9, Lemma (3.1)]. Thus $\left\{t_{1}, t_{2}, t_{3}, t_{4}\right\} \subset$ $T\left(W^{\prime},\{a, b\}\right)$, where $\langle a, b\rangle=\left\langle t_{1}, t_{2}, t_{3}, t_{4}\right\rangle$ and $\left(W^{\prime},\{a, b\}\right)$ is a Coxeter system.

Suppose for the sake of contradiction that $t_{3}<_{T} t_{1}$, then $t_{4}<_{T} t_{3}<_{T} t_{1}<_{T} t_{2}$, since $D((u<y<v))=\emptyset$ and $D((u<x<v))=\{1\}$. Moreover since $t_{1} t_{2}=t_{3} t_{4}$ one has that $t_{4} t_{3} t_{1} t_{2}=e$. On the other hand $\left(W^{\prime},\{a, b\}\right)$ is either a finite or affine Coxeter system, and thus $t_{4} t_{3} t_{1} t_{2} \neq e$ by Theorem 3.7. We have obtained our desired contradiction. The statement $t_{4}<_{T} t_{2}$ is proved in a similar manner.

We can now prove the following proposition.

Proposition 3.9. Let $\Delta$ be the lexicographically-first path in $B_{k}(u, v)$. Then $D(\Delta)=\emptyset$, i.e, $\Delta$ is rising.

Proof. Let $<_{T}$ be a reflection order and let $C=\left(x_{0}=u<x_{1}<\cdots<x_{k}=v\right)$ be the lexicographically-first path in $B_{k}(u, v)$. Let us suppose that $D(C) \neq \emptyset$, and consider the

smallest $i$ such that $x_{i}^{-1} x_{i+1}<_{T} x_{i-1}^{-1} x_{i}$. Let $\left(x_{i-1}<x_{i}^{\prime}<x_{i+1}\right) \stackrel{\text { def }}{=}$ flip $\left(\left(x_{i-1}<x_{i}<x_{i+1}\right)\right)$, and define $C^{\prime}=\left(x_{0}<\cdots<x_{i-1}<x_{i}^{\prime}<x_{i+1}<\cdots<x_{k}\right)$. Proposition 3.8 yields that $x_{i-1}^{-1} x_{i}^{\prime}<_{T} x_{i-1}^{-1} x_{i}$, and so $C^{\prime}$ occurs earlier in the lexicographic order, contradicting the choice of $C$.

Dyer [11, Proposition (4.3)] showed that the lexicographically-first path is the unique rising path in $B_{\operatorname{rk}([u, v])}(u, v)$. On the other hand, the above proposition shows that the lexicographically-first path in non-empty sets $B_{k}(u, v)$ with $k \equiv \operatorname{rk}([u, v])(\bmod 2)$ is rising. We remark that this is the best that can be done to extend Dyer's result for $k \neq \operatorname{rk}([u, v])$, since there can be more than one rising path in $B_{k}(u, v)$; for instance, consider $I_{2}(m)$ with $m>3$.

As an immediate consequence of Proposition 3.9, we have

Corollary 3.10. If $\widetilde{\psi}_{u, v}(\boldsymbol{c}, \boldsymbol{d})$ has a term of degree $k$, then $\left[\boldsymbol{c}^{k}\right]_{u, v}>0$.

In [4], we study the coefficients of degree $k$, where $k$ is the minimum integer with $\left[\mathbf{c}^{k}\right]_{e, w_{0}}>0$ and $w_{0}$ is the longest element of a finite Coxeter group. Further properties of the lowest-degree coefficients of $\widetilde{\psi}_{u, v}(\mathbf{c}, \mathbf{d})$ for some Bruhat intervals are discussed in [5].

Acknowledgements. I thank Louis Billera for suggesting comparing the descentset distribution of the BW-labeling and the reflection order as well as reading (several) earlier versions of this paper. I also thank him for insightful conversations and kind advice throughout the years. I am indebted to the anonymous referee for his valuable comments on how to improve this paper. Of course, all the errors that remain are mine.

\section{References}

[1] Louis Billera and Francesco Brenti. Quasisymmetric functions and Kazhdan-Lusztig polynomials. Israel Journal of Mathematics, 184:317-348, 2011. 10.1007/s11856-0110070-0. 
[2] Anders Björner and Francesco Brenti. Combinatorics of Coxeter groups, volume 231 of Graduate Texts in Mathematics. Springer, New York, 2005.

[3] Anders Björner and Michelle Wachs. Bruhat order of Coxeter groups and shellability. Adv. in Math., 43(1):87-100, 1982.

[4] Saúl A. Blanco. Shortest path poset of finite Coxeter groups. In 21st International Conference on Formal Power Series and Algebraic Combinatorics (FPSAC 2009), Discrete Math. Theor. Comput. Sci. Proc., AK, pages 189-200. Discrete Math. Theor. Comput. Sci., Nancy, 2009.

[5] Saúl A. Blanco. Shortest path poset of Bruhat intervals and the complete cd-index. PhD thesis, Cornell University, 2011.

[6] Francesco Brenti. Combinatorial expansions of Kazhdan-Lusztig polynomials. J. London Math. Soc. (2), 55(3):448-472, 1997.

[7] Paola Cellini. T-increasing paths on the Bruhat graph of affine Weyl groups are self-avoiding. J. Algebra, 228(1):107-118, 2000.

[8] Matthew J. Dyer. Reflection subgroups of Coxeter systems. J. Algebra, 135(1):57-73, 1990.

[9] Matthew J. Dyer. On the "Bruhat graph" of a Coxeter system. Compositio Math., 78(2):185-191, 1991.

[10] Matthew J. Dyer. Hecke algebras and shellings of Bruhat intervals. II. Twisted Bruhat orders. In Kazhdan-Lusztig theory and related topics (Chicago, IL, 1989), volume 139 of Contemp. Math., pages 141-165. Amer. Math. Soc., Providence, RI, 1992.

[11] Matthew J. Dyer. Hecke algebras and shellings of Bruhat intervals. Compositio Math., 89(1):91-115, 1993.

[12] James E. Humphreys. Reflection groups and Coxeter groups, volume 29 of Cambridge Studies in Advanced Mathematics. Cambridge University Press, Cambridge, 1990. 